\title{
Relationship between Lateral Femoral Bowing and Varus Knee Deformity Based on Two-Dimensional Assessment of Side-to-Side Differences
}

\author{
Myung-Rae Cho, MD, Young Sik Lee, MD, and Won-Kee Choi, MD \\ Department of Orthopaedic Surgery, Daegu Catholic University Medical Center, Daegu, Korea
}

\begin{abstract}
Purpose: The objective was to evaluate the relationship between side-to-side differences of lateral femoral bowing and varus knee deformity based on two-dimensional (2D) assessment in unilateral total knee arthroplasty (TKA).

Materials and Methods: A total of 143 patients with varus knee osteoarthritis who underwent unilateral TKA were enrolled. We evaluated the sideto-side differences of the frontal lower limb alignment by assessing lateral femoral bowing, anatomical medial distal femoral angle, and anatomical medial proximal tibial angle (aMPTA).

Results: The average values of all anatomical indices were significantly different between the operated side and the non-operated side ( $<<0.05)$. The side-to-side difference in hip knee ankle (HKA) angle had a statistically significant correlation with that in lateral femoral bowing (intraclass correlation coefficient, $0.259 ; \mathrm{p}=0.002$ ) and that in aMPTA. Linear regression analysis showed $0.199^{\circ}$ of side-to-side difference in lateral femoral bowing was associated with $1^{\circ}$ of side-to-side difference in bilateral HKA angle.

Conclusions: The side-to-side difference in lateral femoral bowing showed a tendency to increase in proportion to varus knee deformity based on $2 \mathrm{D}$ assessment in unilateral TKA patients.
\end{abstract}

Keywords: Knee, Femur, Osteoarthritis, Varus deformity, Bowing

\section{Introduction}

Lateral femoral bowing is often seen in patients with varus knee osteoarthritis (OA), more prominently in Asians. As varus knee OA progresses, the degree of lateral femoral bowing also increases $^{1,2)}$. The clinical problem of lateral femoral bowing is that a several lateral bowing increases the risk of errors in femoral bone cutting during total knee arthroplasty (TKA), which can result in

Received October 10, 2017; Revised (1st) November 9, 2017;

(2nd) December 14, 2017; (3rd) December 20, 2017;

Accepted December 22, 2017

Correspondence to: Won-Kee Choi, MD

Department of Orthopaedic Surgery, Daegu Catholic University Medical Center, 33 Duryugongwon-ro 17-gil, Nam-gu, Daegu 42472, Korea

Tel: +82-53-650-4277, Fax: +82-53-652-4272

E-mail: cwk1009@hanmail.net

Source of funding: This study was supported by Daegu Catholic University.

This is an Open Access article distributed under the terms of the Creative Commons Attribution Non-Commercial License (http://creativecommons.org/licenses/by-nc/4.0/) which permits unrestricted non-commercial use, distribution, and reproduction in any medium, provided the original work is properly cited. functional deterioration after surgery ${ }^{3,4)}$.

Assuming symmetrical features of human anatomy and absence of congenital anatomic differences, it is possible to evaluate radiologically the relationship between side-to-side differences of anatomic indices and the extent of varus knee deformity in a patient with unilateral degenerative changes of the knee.

There are some controversies on the relationship between lateral femoral bowing and varus knee deformity ${ }^{1,5)}$. The objective of this study was to evaluate side-to-side differences of lateral femoral bowing and degrees of varus knee deformity using a two-dimensional (2D) measurement method. We evaluated the differences in coronal alignment femoral lateral bowing between the operated side and the non-operated side in patients who underwent unilateral TKA. The patients had pain on the operated side that interfered with daily life and did not improve in spite of conservative treatment using non-steroidal anti-inflammatory drugs and injection therapy. The non-operated side in same patients did not require TKA due to the absence of pain or pain responsive to conservative treatment. 
We hypothesized that the side with greater varus deformity would show greater lateral femoral bowing deformity than the other side in a patient who underwent unilateral TKA.

\section{Materials and Methods}

This study was conducted in a retrospective manner using the radiological data obtained from 143 patients who underwent unilateral TKA (Fig. 1) performed by the same surgeon from January 2007 to December 2013. During this period, a total of 975 primary TKAs were performed at our institution: bilateral TKA in 389 patients (778 TKAs) and unilateral TKA in 197 patients (197 TKAs).

Of the 197 unilateral TKA patients, those with the following conditions were excluded: valgus knee deformity $(n=22)$, a past history of lower limb mid shaft fracture surgery affecting the mechanical axis of the lower limb $(\mathrm{n}=8)$, and inflammatory arthritis $(n=11)$. Thirteen additional patients were also excluded due to poor preoperative radiological information caused by incorrect postures. The characteristics of the included patients are summarized in Table 1.

The study received Institutional Review Board approval at the author's institution (Daegu Catholic University Hospital, IRB number: CR-16-112).

\section{Radiographic Measurements}

The knees were categorized as the operated side and nonoperated side. The Kellgren-Lawrence (KL) grade of each side was checked by one of the authors using the standing short knee anteroposterior (AP) radiograph obtained before surgery. Two researchers who were blinded to the study assessed the following parameters on the 3-month postoperative standing whole leg AP view using the picture archiving and communication system

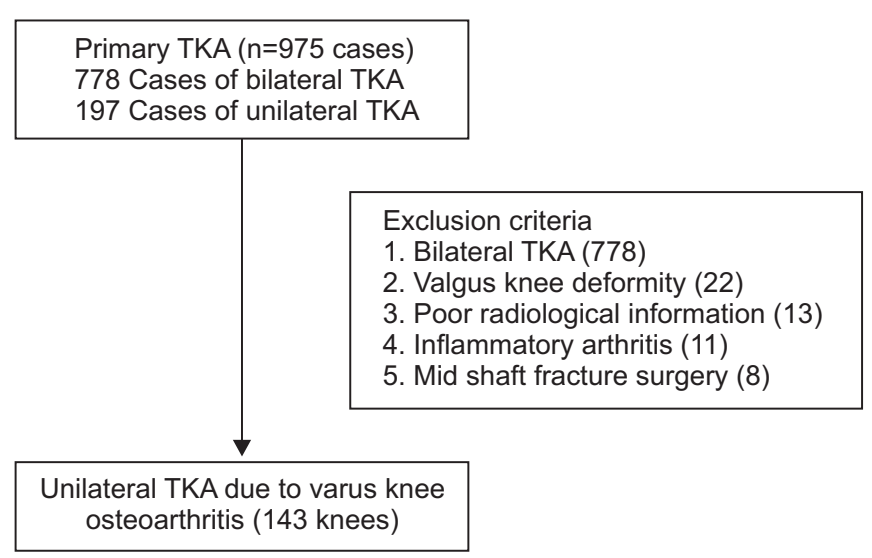

Fig. 1. Flowchart of patient selection. TKA: total knee arthroplasty.
(PACS) twice with an interval of one month: hip knee ankle (HKA) angle, lateral femoral bowing, anatomical medial distal femoral angle (aMDFA), and anatomical medial proximal tibial angle (aMPTA) ${ }^{6}$. The HKA angle was defined as the angle between the line drawn from the center of the femoral head to the midpoint of the tibial eminence (spine) and the line connecting the center of the talus and the midpoint of the tibial eminence (spine). Lateral femoral bowing was defined as an acute angle formed between the line drawn at the center of the femur below the level of the lesser trochanter to pass the center of the femur at a point $5 \mathrm{~cm}$ distal to the starting point and the line extending from the center of the femoral distal condyle through the center of the femur at a $5 \mathrm{~cm}$ proximal portion and a $5 \mathrm{~cm}$ further proximal point ${ }^{3)}$ (Fig. 2A). Lateral bowing was expressed in a positive value while medial bowing was expressed in a negative value. The aMDFA was defined as the medial angle formed by the anatomical femoral axis and the line connecting the distal femoral condyles (Fig. 2B). The aMPTA was defined as the medial angle formed from by the mechanical tibial axis and the bilateral tibial plateau (Fig. 2C).

\section{Comparison of Measured Values}

After comparing the side-to-side differences in anatomical index values in unilateral TKA patients, we investigated the differences according to the KL grade using a paired $t$-test. The as-

Table 1. Demographic and Clinical Data from Patients with Varus Knee Osteoarthritis

\begin{tabular}{lc}
\hline Characteristic & Value \\
\hline Total & 143 \\
Female:male & $112: 31$ \\
Right:left & $65: 78$ \\
Age $(\mathrm{yr})$ & $69.24 \pm 6.79$ \\
KL grade of knees & \\
TKA side & \\
Grade 3 & $56(39)$ \\
Grade 4 & $87(61)$ \\
Non-TKA side & \\
Grade 1 & $73(51)$ \\
Grade 2 & $59(41)$ \\
Grade 3 & $11(8)$ \\
HKA angle $\left(^{\circ}\right)$ & \\
TKA side & $169.99 \pm 4.65$ \\
Non-TKA side & $175.43 \pm 3.73$
\end{tabular}

Values are presented as mean \pm standard deviation or number (\%). KL: Kellgren-Lawrence, TKA: total knee arthroplasty. 

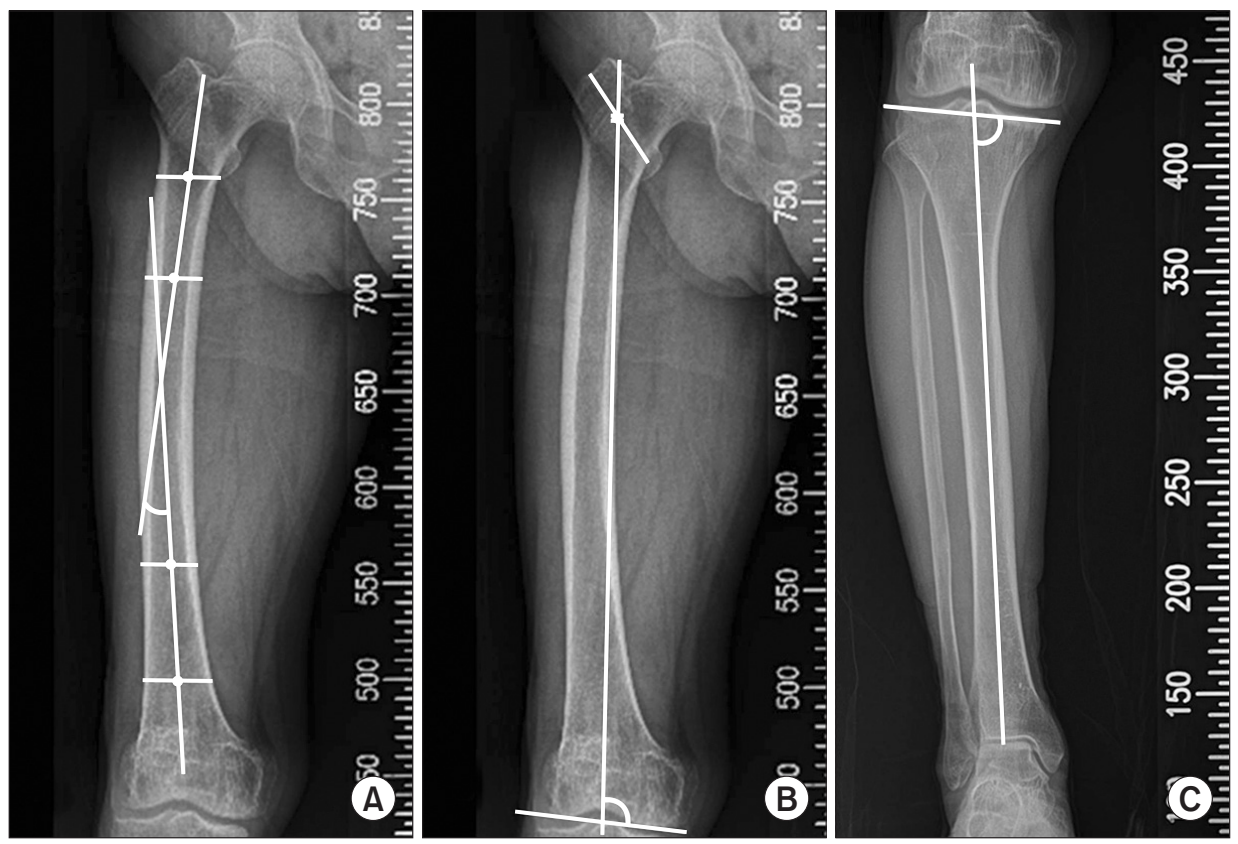

Fig. 2. Radiographic measurements of lateral femoral bowing (A), medial distal femoral angle (B), and medial proximal tibial angle $(\mathrm{C})$.

sociations between the side-to-side differences in HKA angle and those in anatomical indices were evaluated using Pearson correlation coefficient.

\section{Statistical Analysis}

Differences in cross-sectional anatomical indices between the operated side and non-operated side were compared using a paired $t$-test. SPSS ver. 18.0 (SPSS Inc., Chicago, IL, USA) was used for all statistical analysis. A p-value less than 0.05 was considered statistically significant. Pearson correlation coefficient was used to assess the association between the side-to-side difference in HKA angle and that in anatomical indices including femoral lateral bowing. The association between the HKA angle and femoral lateral bowing was determined by obtaining a linear regression line.

\section{Reliability Analysis of Radiation Measurement}

Two researchers who were blinded to the objective of this study performed measurement after receiving training on radiographic measurements. Measurement was carried out by each researcher twice with a one-month interval. After completion of measurement, we analyzed the inter- and intraobserver reliability. Based on the observed reliability, measurements taken by a single investigator were used in the analysis.

\section{Results}

The intraclass correlation coefficients (ICCs) of the radio-
Table 2. Intraclass Correlation Coefficients (ICCs) of Radiographic Measurements

\begin{tabular}{lcc}
\hline \multirow{2}{*}{ Parameter } & \multicolumn{2}{c}{ ICC } \\
\cline { 2 - 3 } & Intraobserver & Interobserver \\
\hline LFB $\left(^{\circ}\right)$ & 0.91 & 0.89 \\
aMDFA $\left(^{\circ}\right)$ & 0.85 & 0.77 \\
aMPTA $\left(^{\circ}\right)$ & 0.88 & 0.84 \\
HKA angle $\left(^{\circ}\right)$ & 0.92 & 0.81 \\
\hline
\end{tabular}

LFB: lateral femoral bowing, aMDFA: anatomical medial distal femoral angle, aMPTA: anatomical medial proximal tibial angle, HKA: hip knee ankle.

graphic measurements were all above 0.8 (Table 2). The mean Lateral femoral bowing was $4.37^{\circ} \pm 4.65^{\circ}$ on the operated side and $3.37^{\circ} \pm 4.68^{\circ}$ on the non-operated side (Table 3 ).

1. Comparison of Anatomic Indices Including Lateral Femoral Bowing between the Operated Side and Non-Operated Side

Based on the paired $t$-test, the average values of anatomical indices showed statistically significant differences between the operated and non-operated sides ( $\mathrm{p}<0.05$ for all) (Table 4). The sample size was 143 patients, which had more than $80 \%$ statistical power for detecting significant side-to-side differences in lateral femoral bowing.

2. Association of Side-to-Side Differences in HKA Angle and Anatomical Indices

To determine the association of side-to-side difference in 
Table 3. Anatomical Indices of the Operated Side and Non-Operated Side in Unilateral Total Knee Arthroplasty Patients

\begin{tabular}{lcc}
\hline \multicolumn{1}{c}{ Parameter } & No. & Mean \pm SD \\
\hline Operated side $\left(^{\circ}\right)$ & & \\
LFB & 143 & $4.37 \pm 4.65$ \\
MDFA & 143 & $95.45 \pm 3.03$ \\
MPTA & 143 & $84.31 \pm 3.47$ \\
Non-operated side $\left(^{\circ}\right)$ & & \\
LFB & 143 & $3.37 \pm 4.68$ \\
MDFA & 143 & $96.14 \pm 3.74$ \\
MPTA & 143 & $86.34 \pm 2.53$ \\
\hline
\end{tabular}

SD: standard devation, LFB: lateral femoral bowing, MDFA: medial distal femoral angle, MPTA: medial proximal tibial angle.

Table 4. Side-to-Side Comparison of Anatomical Indices Including Femoral Lateral Bowing in Unilateral TKA Patients

\begin{tabular}{lrcc}
\hline \multicolumn{1}{c}{ Variable } & Mean \pm SD & 95\% CI & p-value \\
\hline TKA-non TKA side $\left(^{\circ}\right)$ & & & \\
Lateral femoral bowing & $0.99 \pm 3.86$ & 0.35 to 1.63 & 0.00 \\
Medial distal femoral angle & $-0.68 \pm 4.05$ & -1.35 to -0.01 & 0.04 \\
Medial proximal tibial angle & $-2.03 \pm 3.47$ & -2.60 to -1.45 & 0.00 \\
\hline
\end{tabular}

TKA: total knee arthroplasty, SD: standard deviation, CI: confidence interval.

HKA angle with that in anatomical indices, Pearson correlation coefficient was used. The side-to-side difference in HKA angle showed statistically significant association with that in lateral femoral bowing (ICC, 0.259; $\mathrm{p}=0.002$ ) and in MPTA (ICC, 0.534; $\mathrm{p}=0.000)$.

\section{Regression Analyses of Side-to-Side Difference in HKA \\ Angle and Lateral Femoral Bowing}

A linear regression model with $12.4 \%$ of explanatory power showed that there was $0.199^{\circ}$ of side-to-side difference in lateral femoral bowing per $1^{\circ}$ of side-to-side difference in HKA (Fig. 3). For aMPTA, a linear regression model with $46.8 \%$ of explanatory power showed there was $0.389^{\circ}$ of side-to-side difference per one degree of side-to-side difference in HKA.

\section{Discussion}

The most important finding of this study is that lateral femoral bowing shows a tendency to increase proportionally to varus knee deformity in 2D assessment. Previously, some authors evaluated the relationship between knee OA and knee alignment

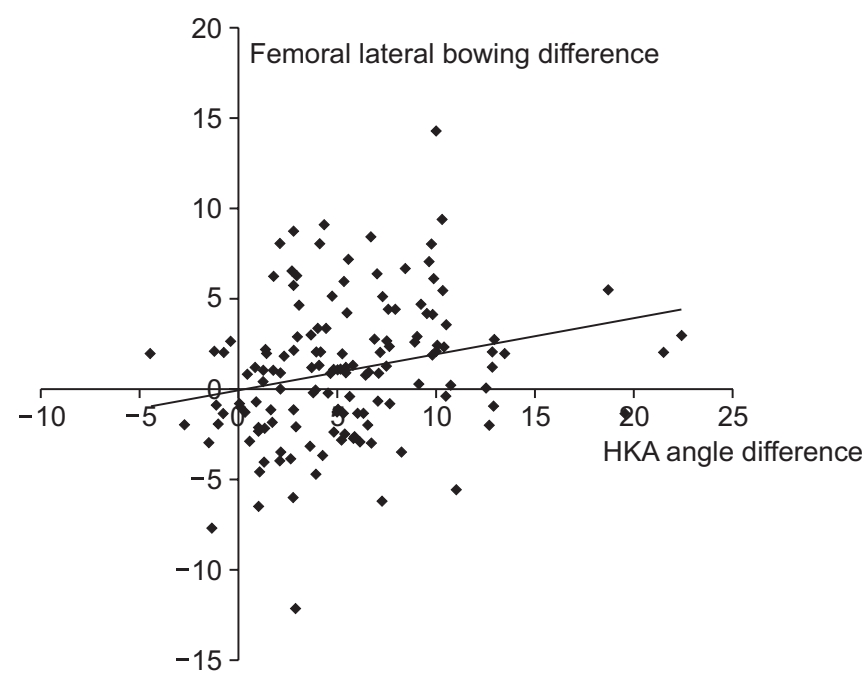

Fig. 3. A linear regression model with $12.4 \%$ of explanatory power showing $0.199^{\circ}$ of side-to-side difference in lateral femoral bowing per $1^{\circ}$ of side-to-side difference in hip knee ankle (HKA) angle.

in a cross-sectional study ${ }^{7}$; however, the knees in the study group and control group were not from the same patients in the study. Therefore, they only investigated the difference of frontal lower limb alignment in accordance with degrees of varus knee deformity. By contrast, we evaluated the relationship between lateral femoral bowing and varus knee deformity by comparing the operated side and non-operated side of the enrolled patients who had unilateral TKA. Thus, we were able to evaluate whether the side-to-side difference in lateral femoral bowing was associated with progression of varus knee deformity.

Lasam et al. ${ }^{3)}$ reported that an average of $5.4^{\circ}$ of lateral femoral bowing was present in a Korean population $(n=367)$ who underwent unilateral TKA due to knee OA. They also found that an average of $3^{\circ}$ of femoral lateral bowing was present in 60 people with normal knees in the age- and gender-matched control group. The measurement method of lateral femoral bowing using PACS in that study was also utilized in our study. In our patients, the mean lateral femoral bowing of the operated side was $4.36^{\circ}$, compared to $3.37^{\circ}$ in the non-operated side. It has been reported that aMPTA has the value of $87^{\circ}$ for the tibial anatomical axis. Also, MDFA has the value of $99^{\circ}$ for the femoral anatomical axis $^{8}$. In our study, the mean anatomical MDFA for the operated side and non-operated side was $95.45^{\circ} \pm 3.03^{\circ}$ and $96.14^{\circ} \pm 3.74^{\circ}$, respectively, whereas the mean anatomical MPTA was $84.31^{\circ} \pm 3.47^{\circ}$ and $86.34^{\circ} \pm 2.53^{\circ}$, respectively.

In this study, side-to-side differences in HKA angle and lateral femoral bowing were shown to have statistically significant association. With a linear regression model that had an explanatory 
power of $12.4 \%$, it was identified that a $0.199^{\circ}$ of side-to-side difference in lateral femoral bowing would occur per one degree difference in HKA angle between two sides.

The association of anatomic side-to-side differences occurring as a secondary change due to the progression of degenerative arthritis with other factors such as gender and age has been reported previously" ${ }^{9}$. In the current study, we only focused on differences in anatomic indices between the knees treated with TKA and those that did not require any surgery.

This study has several limitations. First, bilateral anatomical symmetry around the knee joint has not yet been established. However, side-to-side comparison of knee anatomy seems to be common in unilateral joint disease studies ${ }^{10-12)}$. Eckstein et al. ${ }^{12)}$ showed comparable bilateral knee joint anatomy in terms of the articular surface area and volume and the local thickness of cartilage using magnetic resonance imaging. Second, there is a possibility of radiographic measurement errors. In the case where surgery is required due to severe degenerative arthritis, limitation of knee extension and rotational changes may cause measurement errors. In order to address this issue, one of the authors screened the whole leg standing views of patients who underwent TKA for unilateral varus knee arthritis prior to measurement and excluded 13 patients from the study, who showed differences in the knee joint level due to the limitations of extension, inadequate postures in radiography, or differences in rotation with differently sized lesser trochanters. Third, this study was focused on anatomic changes in 2D coronal alignment only. Recently, many studies have discussed anatomic changes using three-dimensional (3D) spatial images ${ }^{13)}$. Akamatsu et al. ${ }^{14)}$ reported 3D measurements of lateral femoral bowing were much smaller than $2 \mathrm{D}$ measurements. They also suggested that computed tomography is more useful for assessing lateral femoral bowing when planning reconstructive knee surgeries. Fourth, though lateral femoral bowing could cause bone cutting errors in artificial joint surgeries depending on the degree of varus knee deformity ${ }^{3,4}$, we did not evaluate its impact on the postoperative clinical results, which was beyond the scope of the study, and thus this should be taken into consideration when interpreting our results.

\section{Conclusions}

Lateral femoral bowing showed a tendency to increase proportionally to varus knee deformity in $2 \mathrm{D}$ assessment.

\section{Conflict of Interest}

No potential conflict of interest relevant to this article was reported.

\section{References}

1. Mullaji AB, Marawar SV, Mittal V. A comparison of coronal plane axial femoral relationships in Asian patients with varus osteoarthritic knees and healthy knees. J Arthroplasty. 2009; 24:861-7.

2. Tang WM, Zhu YH, Chiu KY. Axial alignment of the lower extremity in Chinese adults. J Bone Joint Surg Am. 2000;82: 1603-8.

3. Lasam MP, Lee KJ, Chang CB, Kang YG, Kim TK. Femoral lateral bowing and varus condylar orientation are prevalent and affect axial alignment of TKA in Koreans. Clin Orthop Relat Res. 2013;471:1472-83.

4. Yau WP, Chiu KY, Tang WM, Ng TP. Coronal bowing of the femur and tibia in Chinese: its incidence and effects on total knee arthroplasty planning. J Orthop Surg (Hong Kong). 2007;15:32-6.

5. Li YR, Gao YH, Qi X, Liu JG, Ding L, Yang C, Zhang Z, Li $\mathrm{SQ}$. Analysis of factors that affect the precision of the radiographic lateral femoral bowing angle using a three-dimensional computed tomography-based modelling technique. J Orthop Surg Res. 2017;12:92.

6. Sabharwal S, Zhao C, Edgar M. Lower limb alignment in children: reference values based on a full-length standing radiograph. J Pediatr Orthop. 2008;28:740-6.

7. Tanamas S, Hanna FS, Cicuttini FM, Wluka AE, Berry P, Urquhart DM. Does knee malalignment increase the risk of development and progression of knee osteoarthritis? A systematic review. Arthritis Rheum. 2009;61:459-67.

8. Hsu RW, Himeno S, Coventry MB, Chao EY. Normal axial alignment of the lower extremity and load-bearing distribution at the knee. Clin Orthop Relat Res. 1990;(255):215-27.

9. Bastick AN, Belo JN, Runhaar J, Bierma-Zeinstra SM. What are the prognostic factors for radiographic progression of knee osteoarthritis? A meta-analysis. Clin Orthop Relat Res. 2015;473:2969-89.

10. Dargel J, Feiser J, Gotter M, Pennig D, Koebke J. Side differences in the anatomy of human knee joints. Knee Surg Sports Traumatol Arthrosc. 2009;17:1368-76.

11. Anderson AF, Snyder RB, Federspiel CF, Lipscomb AB. Instrumented evaluation of knee laxity: a comparison of five 
arthrometers. Am J Sports Med. 1992;20:135-40.

12. Eckstein F, Muller S, Faber SC, Englmeier KH, Reiser M, Putz R. Side differences of knee joint cartilage volume, thickness, and surface area, and correlation with lower limb dominance: an MRI-based study. Osteoarthritis Cartilage. 2002;10:914-21.

13. Duparc F, Thomine JM, Simonet J, Biga N. Femoral and tibial bone torsions associated with medial femoro-tibial os- teoarthritis: index of cumulative torsions. Orthop Traumatol Surg Res. 2014;100:69-74.

14. Akamatsu Y, Kobayashi H, Kusayama Y, Kumagai K, Saito T. Femoral shaft bowing in the coronal and sagittal planes on reconstructed computed tomography in women with medial compartment knee osteoarthritis: a comparison with radiograph and its predictive factors. Arch Orthop Trauma Surg. 2016;136:1227-32. 